Session 2273

\title{
BME GOES TO THE MOVIES: DEVELOPING ETHICAL PERSPECTIVE IN BIOENGINEERS
}

\author{
S. Brophy, K. Bliley, A. Gray, C. Mathieson, E. Mowry, J. Collins \\ Department of Biomedical Engineering, Vanderbilt University, Nashville, TN 37235
}

\begin{abstract}
As bioengineering students enter their new profession they must become sensitive to the ethical ramifications of their work. Bioengineering educational programs seek opportunities to engage students in the exploration of ethical dilemmas in bioengineering. Our involvement with the VaNTH ERC and the learning sciences' principles that guide their design of instruction has provided us with insights into potential methods to develop ethical awareness in our students. We are exploring the use of movies as a vehicle for identifying and refining understanding of ethical issues in a case. Although classroom discussions around case studies sometimes engage students, often the homogeneity and inexperience of the class result in a narrow focus on issues. In order to overcome this instructional problem, we have identified several popular movies that raise issues ranging from public health to research methods and practice. Students in a $2^{\text {nd }}$-year undergraduate course on thermodynamics of biological attend an informal viewing of a movie, as one of their optional assignments (required as part a participation grade). Also invited were students from other disciplines on campus. This learning activity began by students generating their initial thoughts about ethical issues that relates to a movie they were about to watch. After watching the movie, everyone is asked to fill out a short questionnaire where they record issues they notice in the movie and potential ethical ramifications. Then, an open discussion follows in an effort to identify multiple perspectives. In this first study we focus on issues related to human subjects in medical research. Students view "Miss Evers' Boys" as a catalyst for thinking about relevant issues. One of the goals of this instructional method is to prepare students to investigate the details of these issues identified in the Belmont Report ${ }^{2}$. We find that individually students can identify several relevant issues both before and after, but not all the issues. The group as a whole can identify many aspects of the principles for "Respect for Persons" and "Beneficence", but miss issues related to "Justice". This instructional method has the potential for demonstrating the important issues related to conducting research involving human subjects. This papers discusses the benefits of using movies for instruction, describes a short interventions we designed around watching a movie and a short description of impact of these results on instruction.
\end{abstract}




\section{Introduction}

The rapidly growing field of bioengineering is opening a range of possibilities for advancing science and increasing the quality of life for many people. Unfortunately, the number of ethical issues grows with each new innovation. Our engineering students will need to continually be sensitive to how their work contributes to the advancement of science, how the science is conducted and how society is affected by these new innovations. The issues are not always clear-cut and often can be difficult to recognize in real world situations. Therefore, it is not surprising that core competency in ethics is an ABET requirement. The Internet has a large amount of sample cases and suggestion to assist an instructor in fulfilling this requirement (for example see http://onlineethics.org/reseth/mod/biores.html for materials specific to bioengineering). Many instructors use cases studies to help students notice the relevant features of an ethical dilemma. Also, using a framework similar to models of problem solving, students can learn methods for systematically analyzing these cases to determine when they should look for more information and what are the ramifications of various decisions.

One of the most challenging aspects of analyzing an ethical problem is the initial identification of the problem. However, when students study ethics in a course, they are usually doing it as a specific part of the course. They are given cases to analyze and they actively seek to find factors in a sample case that could potentially contain ethical elements. The process of providing cases to study ethics could limit the opportunities to develop their own skills for identifying relevant ethical cases. This is a very important and difficult step and should be part of instruction. Therefore, a valuable addition to instruction would be to create opportunities for students to identify potential ethical issues from a wider context that might imply multiple none related ethical issues.

We are conducting a series of studies to explore the potential of using movies as instructional tools for ethics. The central goal of this study is to explore the potential of using commercial movies as a catalyst for identifying ethical issues and as a catalyst for a discussion starter. Future studies focus on how these movie activities can be built on during classroom discussions around cases related to the original movie.

\section{Using Movies as Catalysts for Noticing}

Movies and videos have many instructional benefits when set in the right context $(\mathrm{CTGV}, 1997)^{3}$. The Cognition and Technology Group at Vanderbilt (CTGV) have used video based challenges as a mechanism for helping students manage complex mathematical problems. One of the major benefits of using videos is the context it provides for how the knowledge students are learning apply in real situations. Often the abstract presentation of ideas and concepts fail to provide clues for the conditions for when to apply new knowledge (Bransford, et. al. 2000) ${ }^{4}$. Therefore, students could view a movie to observe on example of the conditions that govern the application of specific knowledge. The CTGV find have noted that instruction that uses only one context does not provide students with enough of a context to generalize across multiple context. Therefore, student need to experience multiple context in order to begin to understand subtle difference between concepts. Therefore, we would like to begin instruction with 
an informal activity around a movie, then exam various cases to understand the multiple dimensions of an ethical issue.

Movies can be very engaging and appeal to a large audience. The richness of the plot can raise a level of complexity that learners need to manage and try to comprehend. In addition, movies can provide an interesting account of historical events that can be very educational to bioengineering students. For example, the movie called "Miss Evers' Boys" (1997) ${ }^{1}$ is a drama based on the Tuskegee study started in 1932 . This four-decade research experiment was designed to study but not treat syphilis in the black men of Alabama. Funding ran out before the men could be treated, so the study continued but only to document the effects of the disease on black men. The movie raises interesting issues of how to perform clinical trials and the rights of patients. Another example is "The Insider", a story about an insider in the tobacco industry who blows the whistle on the clandestine affairs of the cigarette manufacturers. This film raises many issues ranging from personal choice, health of non smokers due to incidental smoke, and reporting of information by the media to the mass public. These movies are great illustrations of the kinds of decisions people make, why they made them and the ramifications of these decisions. Studying these movies as part of a course can provide students with the opportunity to identify and discuss the complex relationship of factors related to ethical situations. We believe that through discussions with a diverse group of students a range of perspectives can be raised and explored. The result is a learning experience that leads to a better understanding of the range of ethical issues.

\section{Obtaining Multiple Perspectives Through Discussion}

Another important aspect of analyzing an ethical case is defining the multiple perspectives of a case. Even if instruction on how to approach an ethical dilemma is integrated into engineering students course of study, it is quite possible that students will only hear perspectives from those who have a similar background. Therefore, an addition to the instructional process would be for students to hear perspectives of students from other disciplines. We are exploring the potential of having group discussions by a wide range of a university population after viewing a movie that contains multiple ethical issues. This experience could be a great learning opportunity for all who participate.

\section{Selection Criteria for Movies}

The authors of this paper met to identify several potential movies to use for this series of studies. As a group we listed several possible candidates for movies that contained ethical dilemmas related to engineering, medical or biology issues. Gray took the lead to identify additional movies that fit this role. The group met again to review the list and finalize the selection criteria. We chose to consider movies that depicted real contemporary topics rather than science fiction or futuristic prediction (e.g. the Matrix ${ }^{5}$ ). The other major decision was to select movies that raised several ethical issues. The list was narrowed to three movies, "Miss Evers' Boys" (1997) 1 " "And the Band Played On" (1993, based on a book by Shilts $(1987)^{6}$ and the "The Insider" (1999) ${ }^{7}$. In additional to the original criteria we identified a feature of each of these films that could add an 
interesting dimension to our discussions. Each movie deals with issues of who should pay for damages and who should pay for treatment. Finally, we selected movies that identified multiple links to other issues in current events.

Miss Evers' Boys was selected as our first movie because of its relevance to the Belmont Report. A couple of the authors are involved in a parallel study related to instruction on human subjects in research (Bird, et. al, in progress) ${ }^{8}$. The learning objective involved in Bird's study is to learn how to apply the guidelines and principles defined in the Belmont report to several case studies. The Belmont report identifies three relevant principles to the involvement of human subjects in research: "the principle of respect for persons, beneficence and justice". Miss Evers Boys raises issues about each one of these principles. Therefore, we decided to use this movie as an introduction to these ideas, which will be explored in more depth in the classroom as part of Bird's research.

\section{Method}

In this first study a within subjects design was used to explore effects of using a movie to stimulate students' noticing of relevant ethical issues raised during a movie. The participants in this study consisted of students $(\mathrm{N}=5)$ from a $2^{\text {nd }}$-year undergraduate course on thermodynamics of biological systems. They must attend the movie as part of their participation grade (students must attend at least one of several extracurricular learning activity). Last semester the instructor (Collins) lead class discussions on several ethical cases selected from a variety of topic areas, including stem cell research, scientific reporting, the high cost of development of medical technology, and educational and environmental responsibilities of large corporations.

Other students were invited to attend this movie event. The movie event was advertised using fliers posted around campus inviting people to participate in watching the movie and participate in the discussion (one second year student from Child Development attended, and 1 first year and 2 senior biomedical engineering students attended). The event was scheduled for a Wednesday evening and pizza was served as part of a pre movie social event.

Prior to viewing the movie, students filled out a short survey. In all cases students responses remained anonymous. The first part of the survey contains several questions about the demographics of the audience (e.g. major, enrollment in thermodynamics class, gender, prior coursework in ethics and philosophy). Next, students answered these questions, 1) "If you were to teach a scientist about how to protect a participant in their medical research, what would you tell them?", 2) What rules could you define that would help a medical researcher evaluate their protocol for potential risks to the participants?", 3) If you were a participant in a research study related to your physical health, what kinds of questions would you ask the researcher?"

After watching the movie, students were asked to reflect back on their initial thoughts on the three questions from above. They were given prompts, such as "Look back on what you thought you could teach someone about how to protect a participant in a medical research study. What could you add after seeing the movie? What thoughts did you mention that you now think are not quite correct?" 
Next, $4^{\text {th }}$ year biomedical engineering students lead a 20 minute discussion on ethical issues related to Miss Evers' Boys and beyond. Brief sets of field notes were taken to document this interaction. The $4^{\text {th }}$ year bioengineering students prepared several questions to initiate discussion. The first question they asked targeted the decisions the researchers made in the Tuskegee study and how would you feel in this situation. Next, the audience was asked about their involvement in research studies and any details related to how they felt as a participant. Finally, the discussion lead to issues of liabilities resulting from taking risks with the lives of the participants in the study.

Next, students record any changes in their initial thoughts after the discussion, similar to what they did after viewing the movie. They are given the same set of prompts to stimulate their thinking about the topic.

Finally, students completed a short 3 question survey about their experience with this instructional method. They are asked to rate how closely each statement relates to their experience during this learning activity. Ratings are given using a 5 point scale where $1=$ strongly Disagree and $5=$ Strongly Agree. Students were asked to respond to these statements - "I felt watching the movie helped me notice more issues related to asking people to participate in medical research than before tonight's event", "I felt the discussion gave me additional perspectives I would not have considered on my one" and "I would participate in another movie and discussion even in the future." Also, they are asked to provide any additional comments.

\section{Results}

A small group of undergraduates participated in this initial study. The majority of students participating are from biomedical engineering $\left(\mathrm{N}=8,1^{\text {st }}\right.$ year $=1,2^{\text {nd }}$ year $=5,4^{\text {th }}$ year $=2$ ) and one person from child development $\left(2^{\text {nd }}\right.$ year $)$. Only two of the students have taken a course in ethics or philosophy. None of the participants have seen the movie before.

The questionnaire is designed to identify the quantity and quality of responses students can generate at various times throughout the instructional activity. On average, individual participants generated 1 to 2 of principles for each phase of the intervention. Table 1 summarizes the average ideas students generated.

Table 1 - Average number of concepts generated per questions

\begin{tabular}{|c|l|c|c|c|}
\hline \multicolumn{2}{|l|}{ Question } & \multicolumn{2}{|c|}{ Average Number of Ideas Generated } \\
\cline { 3 - 4 } & $\begin{array}{l}\text { Before } \\
\text { movie }\end{array}$ & $\begin{array}{l}\text { Additional } \\
\text { Ideas After } \\
\text { movie }\end{array}$ & $\begin{array}{l}\text { After } \\
\text { discussion }\end{array}$ \\
\hline 1 & $\begin{array}{l}\text { If you were to teach a scientist about how to protect a } \\
\text { participant in their medical research, what would you } \\
\text { tell them? }\end{array}$ & 2.1 & 1 & 0.1 \\
\hline 2 & $\begin{array}{l}\text { What rules could you define that would help a medical } \\
\text { researchers evaluate their protocol for potential risks to } \\
\text { the participants? }\end{array}$ & 1.2 & 1 & 0.1 \\
\hline 3 & $\begin{array}{l}\text { If you were a participant in a research study to your } \\
\text { physical health, what kinds of questions would you } \\
\text { ask the researcher? }\end{array}$ & 1.5 & 2 & 0.6 \\
\hline
\end{tabular}


Table 2 Response to questions Pre/Post watching the movie

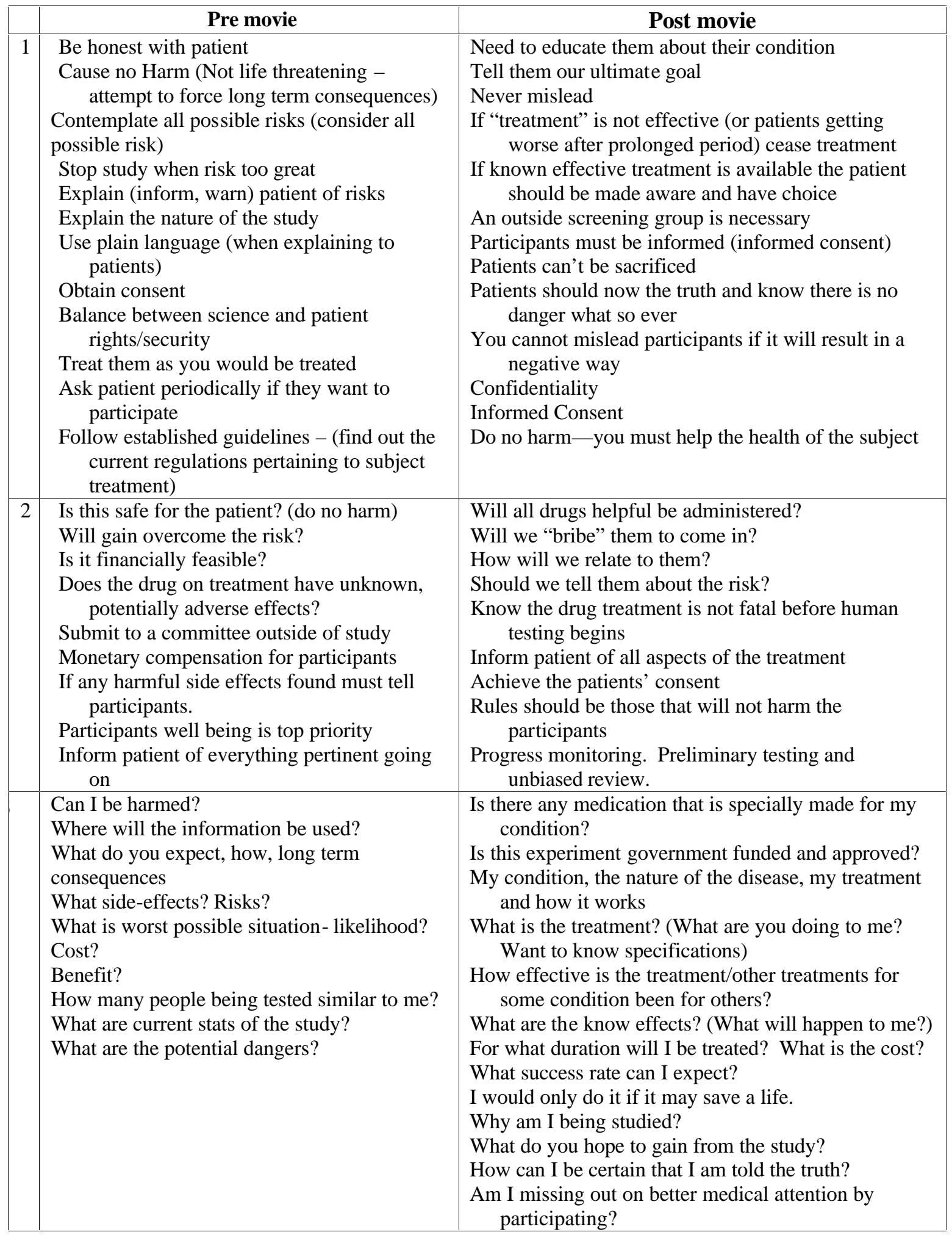


Table 2 illustrates the range of responses students generated at various stages of the intervention. The collection of all the responses from the group for both the pre and post movie watching covered many of the principles highlighted by the Belmont report (ie, do no harm, beneficence, and justice). As Table 1 illustrated each student only contributed on or two ideas either pre or post.

The discussion activity resulted in the generation of a few additional ideas by several members of the group. Prior to the discussion the students generated their own thoughts about the question. The discussion was the first collaborative effort made to share and expand ideas. Table 3 shows several additional comments individuals made after the general discussion.

\section{Table 3- Responses to questions after discussion}

\begin{tabular}{|l|l|}
\hline 1 & Make sure you're funded properly. \\
\hline 2 & $\begin{array}{l}\text { Make sure you know all the effects. Must make sure the subjects are fully aware } \\
\text { of what is going on and are giving their full consent. }\end{array}$ \\
\hline 3 & $\begin{array}{l}\text { What steps will be taken if I experience an adverse effect } \\
\text { What kinds of funds/compensation involved? } \\
\text { Proper funding for the full term of the study? } \\
\text { How will I be compensated if something should go wrong? }\end{array}$ \\
\hline
\end{tabular}

\section{Discussion}

The results of this study illustrate the potential benefits of one instructional method for helping students learn how to identify ethical issues from real world examples and become aware of multiple perspective related to issues close to their profession. Although the number of participants is small $(\mathrm{N}=9)$, there are interesting trends that illustrate both what the group and the individual can achieve during this mild learning intervention using movies.

Prior to watching the movie "Miss Evers' Boys" the group as a whole could generate quite a long list of principles for protecting the rights of the human participants in a research study. The students clearly recognized the importance of "do no harm", and minimize the risk to the participants. Several other students included a discussion about the importance of balancing the risk of the participants with the advancement of science to be gained through the research study. Before watching the movie each student only identified a few ideas for each of the three questions related to research involving human subjects . Interestingly, as a group they identify many of the fundamental ideas of the Belmont Report including issues of "Respect for persons" (e.g. "Treat them as if you were the one having research done on them", "warn them of risk"), "Beneficence (e.g. "Do no harm", "balance between scientific or medical advance and patient rights/security"). However, no indication was made about the principle of "Justice". In fact, no one made reference to anything about the selection criteria for participants in a research study.

After watching the movie almost every student was able to generate at lease 1 or 2 additional ideas for each of the three questions. Therefore, the movie does have some positive impact on helping students notice additional issues. In addition, viewing the 
movies appears to help students identify the need to monitor the progress of treatment and be willing to change treatment as science evolves. Also, several students note the role of the professional researcher and the need to follow established guidelines and to act responsibly. Finally, several students began to pick up on the idea of fiscal responsibility. After watching the movie several students mentioned the need to ensure that the funding will continue through the expected treatment time and if something goes wrong with the treatment will care continue to be given. However, again no mention is made related to issues of "Justice" even though this was a critical point of "Miss Evers Boys". This could be because the survey does not do provide a good catalyst for identifying this as an issue. Alternatively, "justice" could be a difficult concept to recognize without explicitly reflecting on it as part of preparing a research protocol for medical research.

In this study the discussion activity has little impact on changing students' answers on the questionnaire. Only a few students indicate they recognized additional issues as a result of the discussion. The issues the student raise target the financial aspects associated with completing the treatment or compensating participants when the research does not go as intended. It should be noted that the original goal of the discussion was not organized to systematically highlight issues related to the Belmont report. The initial discussion activity was designed to be an informal exploration of ideas generate the multiple perspective provides by a diverse audience. However, the diversity of our audience was not obtained, therefore, this particular objective for our study will be postponed until the next movie event.

Finally, all of the students who responded to the exit survey either agreed or strongly agreed that they learned from watching the movie, they found the event worthwhile and would like to do it again. In students written comments they report that the movie was a great choice and the event was well organized.

\section{Conclusion - Implications for Instruction}

Watching a movie as part of ethics instruction has some great potential benefits. Prior to viewing Miss Evers Boys ${ }^{2}$ individual student can identify a small set of ideas related to issues identified in the Belmont Report (an important document for anyone interested in doing research with human subjects). Watching the movie helped individual student expand on their initial thoughts, which is very powerful. Also, the group as a whole generated an impressive list of issues without even watching the movie. This indicates that the diversity of this small group has the potential of engaging in a discussion where peers can learn many of the issues identified without ever watching the movie (Table 2 illustrates a range of ideas this population can generate with minimal instructional intervention than viewing a movie).

However, the students do not mention the principle of "Justice" (selection of participants) in any of the written or oral reports. This may indicate that "justice" is a difficult concept to understand and requires special consideration during other forms of instruction. The movie itself provides and excellent example of "justice". Therefore, simply making this idea explicit through discussion may be all students need to make the connection. Unfortunately, the small group size and the homogeneity of the group limited our potential to explore the concept that peers from other discipline could provide 
additional insight that could have increase the range of responses reported in Table 2. We will need to conduct additional studies to explore this hypothesis.

This study is the first in a series of studies on ethics instruction in biomedical engineering. We intend to host several more movie and discussion events in the near future. Also, we are going to explore the benefits of extending this study into a second study that explore the benefits of participating in this study, then participating in a classroom discussion around several additional cases related to research with human subjects. We anticipate that this movie going experience will increase students' ability to notice and discuss relevant issues around a case.

\section{Acknowledgements}

This work was supported primarily by the Engineering Research Centers Program of the NSF under Award Number EEC-9876363.

\section{BIBLIOGRAPHIC INFORMATION}

1. Sargent, J. (1997) Miss Ever's Boys. [Movie] Time Warner Entertainment.

2. Belmont Report.(1979) Ethical Principles and Guidelines for the Protection of Human Subjects of Research http://ohrp.osophs.dhhs.gov/humansubjects/guidance/belmont.htm

3. Bransford, J. D., Brown, A. L., \& Cocking, R. R. (Eds.) (2000) How people learn: Brain, mind, experience, and school, Expanded Edition. Washington, DC: National Academy Press. http://www.nap.edu/html/howpeople1/ .

4. Cognition and Technology Group at Vanderbilt. 1997 The Jasper Project: Lessons in Curriculum, Instruction, Assessment, and Professional Development. Mahwah, NJ: Erlbaum..

5. Wachowski, A. (1999). The Matrix.[Movie] Warner Brothers.

6. Spottiswoode (1993). The Band Played On. [Movie] Home Box Office.

7. Roth, T. \& Mann, M. (1999) The Insider. [Movie] Touchstone.

8. Bird, S. (in progress). Human subjects module. Massachusetts Institute of Technology. Cambridge, Massachusetts. 


\title{
BIOGRAPHICAL INFORMATION
}

\section{SEAN P. BROPHY}

Sean P. Brophy received his B.S. degree in Mechanical Engineering from the University of Michigan, an MS in Computer Science from DePaul University, and a PhD in Education and Human Development from Vanderbilt University. Dr. Brophy works with the Learning Technology Center at Vanderbilt to apply current theories of Learning Science to improve instruction at various educational levels. He currently is an Assistant Research Professor in the Department of Biomedical Engineering at Vanderbilt. His current research interests relate to using simulations and models to facilitate students understanding of difficult concepts within engineering as part of the VaNTH Engineering Research Center (ERC).

\section{KARA BLILEY}

Kara Bliley is currently a senior majoring in Biomedical Engineering at Vanderbilt University. She serves as the president of Biomedical Engineering Society, and hopes to increase the involvement of BMES members in activities with the ERC. Her future plans involve obtaining a Ph.D. in Biomedical Engineering.

\begin{abstract}
AMY GRAY
Amy Gray is a recent graduate in biomedical engineering at Vanderbilt University.

\section{CRISTINA MAHTIESON}

Cristina Mahtieson is a student in the Department of Biomedical Engineering, Vanderbilt University, Nashville, TN. In summer 2001 she served as an NSF Research Experience for Undergraduates summer intern in bioethics at the Massachusetts Institute of Technology. Ms. Mathieson plans a career in biomedical engineering with an emphasis in research on female reproductive diseases.
\end{abstract}

\section{EMILY R. MOWRY}

Emily R. Mowry is a rising senior in the Vanderbilt University School of Engineering (VUSE). She has completed several internships in both the VUSE and the Vanderbilt University Medical Center (VUMC), including research in molecular biology, microbiology/immunology, and intracellular engineering. In addition to her contributions on this research, she plans to work with VaNTH-ERC on improving instruction in the undergraduate BME curriculum.

\section{JERRY COLLINS}

Jerry Collins is a member of the biomedical engineering faculty at Vanderbilt University, Nashville, TN. He is industrial liaison for the NSF-sponsored VaNTH Engineering Research Center in bioengineering educational technologies. Dr. Collinsís research interests have included microvascular transport and autonomic control. In addition to his membership in ASEE, he is a member of the College of Fellows of the American Institute of Medical and Biological Engineering, a member of the American Physiological Society and the American Association for the Advancement of Science, and is a board member of the Tennessee Biotechnology Association and the Tennessee Biomedical Engineering Consortium. He is a senior member of the Biomedical Engineering Society and has served the BMES as a member of the Board of Directors, as chair and member of the Interface with Industry Committee, and as editor of the BMES Bulletin from 1991-2001. 